\title{
Bond Directional Anapole Order in a Spin-Orbit Coupled Mott Insulator $\operatorname{Sr}_{2}\left(\operatorname{Ir}_{1-x} \operatorname{Rh}_{x}\right) \mathbf{O}_{4}$
}

\author{
H. Murayama, ${ }^{1}$ K. Ishida $\odot,{ }^{2}$ R. Kurihara, ${ }^{1}$ T. Ono, ${ }^{1}$ Y. Sato, ${ }^{1}$ Y. Kasahara, ${ }^{1}$ H. Watanabe, ${ }^{1}$ Y. Yanase,${ }^{1,4}$ \\ G. Cao, ${ }^{3}$ Y. Mizukami $\odot,{ }^{2}$ T. Shibauchi $\odot,{ }^{2}$ Y. Matsuda $\odot,{ }^{1}$ and S. Kasahara ${ }^{1}$ \\ ${ }^{1}$ Department of Physics, Kyoto University, Kyoto 606-8502 Japan \\ ${ }^{2}$ Department of Advanced Materials Science, University of Tokyo, Chiba 277-8561, Japan \\ ${ }^{3}$ Department of Physics, University of Colorado at Boulder, Boulder, Colorado 80309, USA \\ ${ }^{4}$ Institute for Molecular Science, Okazaki 444-8585, Japan
}

(Received 5 August 2020; revised 3 November 2020; accepted 4 December 2020; published 2 February 2021)

\begin{abstract}
An anapole state that breaks inversion and time-reversal symmetries while preserving translation symmetry of an underlying lattice has aroused great interest as a new quantum state, but only a few candidate materials have been reported. Recently, in a spin-orbit coupled Mott insulator $\operatorname{Sr}_{2}\left(\operatorname{Ir}_{1-x} \mathrm{Rh}_{x}\right) \mathrm{O}_{4}$, the emergence of a possible hidden-order phase with broken inversion symmetry has been suggested at $T_{\Omega}$ above the Néel temperature by optical second-harmonic-generation measurements. Moreover, polarized neutron diffraction measurements revealed broken time-reversal symmetry below $T_{\Omega}$, which was supported by subsequent muon spin relaxation experiments. However, the nature of this mysterious phase remains largely elusive. Here, we investigate the hidden-order phase through the combined measurements of the inplane magnetic anisotropy with exceptionally high-precision magnetic torque and the nematic susceptibility with elastoresistance. A distinct twofold in-plane magnetic anisotropy along the [110] Ir-O-Ir bond direction sets in below about $T_{\Omega}$, providing thermodynamic evidence for a nematic phase transition with broken $C_{4}$ rotational symmetry. However, in contrast to the even-parity nematic transition reported in other correlated electron systems, the nematic susceptibility exhibits no divergent behavior towards $T_{\Omega}$. These results provide bulk evidence for an odd-parity order parameter with broken rotational symmetry in the hidden-order state. We discuss the hidden order in terms of an anapole state, in which the polar toroidal moment is induced by two current loops in each $\mathrm{IrO}_{6}$ octahedron of opposite chirality. Contrary to the simplest loop-current pattern previously suggested, the present results are consistent with a pattern in which the intra-unit cell loop current flows along only one of the diagonal directions in the $\mathrm{IrO}_{4}$ square.
\end{abstract}

DOI: 10.1103/PhysRevX.11.011021

\section{INTRODUCTION}

The search for novel types of ordered states is one of the most exciting and challenging issues of modern condensedmatter physics. In strongly correlated electron systems, charge, spin, and orbital degrees of freedom generate a rich variety of ordered states. Any of these ordered states can be characterized by their behaviors under space-inversion (parity) and time-reversal operations. Among systems with broken parity, toroidal states, in which translational symmetry of the underlying lattice is preserved, have been widely discussed [1,2]. There are two types of toroidal states-axial and polar-where time-reversal symmetry is preserved and broken, respectively [3-5]. An example of the axial toroidal states are the electric-toroidal systems

Published by the American Physical Society under the terms of the Creative Commons Attribution 4.0 International license. Further distribution of this work must maintain attribution to the author(s) and the published article's title, journal citation, and DOI.
Subject Areas: Condensed Matter Physics

Strongly Correlated Materials with a vortexlike arrangement of electric dipoles [6-8]. The order parameter of this state is represented by the electrictoroidal dipole moment $\boldsymbol{\Omega}_{\boldsymbol{A}}$, as illustrated in Fig. 1(a). The polar toroidal state is realized in magneto-toroidal systems with a vortexlike arrangement of spin and loop currents, as illustrated in Figs. 1(b) and 1(c), respectively. The order parameter of these polar toroidal systems is represented by the polar magnetic toroidal dipole moment $\boldsymbol{\Omega}_{\boldsymbol{p}}$. The emergence of the polar toroidal dipole moment has aroused significant interest [9-11]. In particular, the state, in which the polar toroidal dipole is induced by persistent loop currents as illustrated in Fig. 1(c), is a new quantum state of matter, and finding it has been a longstanding quest in condensed matter physics. To distinguish two polar states, as shown in Figs. 1(b) and 1(c), we call the polar toroidal state caused by the loop currents an anapole state. The order parameter of the anapole state is represented by the anapole vector $\boldsymbol{\Omega}_{\boldsymbol{P}} \propto \int \boldsymbol{r} \times \boldsymbol{m}(\boldsymbol{r}) d^{3} r$, where $\boldsymbol{r}$ and $\boldsymbol{m}$ are the position and magnetic moment induced by orbital loop currents, respectively. For ferrotoroidal coupling in a tetragonal system, where $\boldsymbol{\Omega}_{\boldsymbol{A}}$ or $\boldsymbol{\Omega}_{\boldsymbol{P}}$ is aligned parallel to the $a b$ 


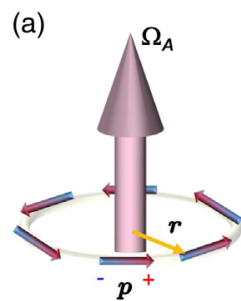

Axial toroidal

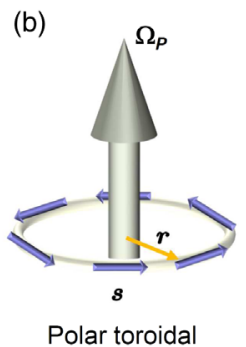

Polar toroidal

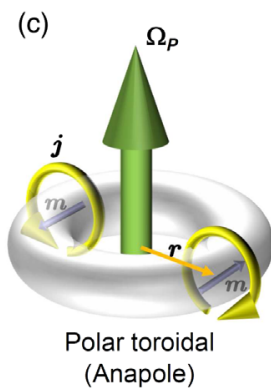

(Anapole)
FIG. 1. Toroidal states with broken inversion symmetry. (a) Electric-toroidal state with a vortexlike arrangement of electric dipoles $\boldsymbol{p}$. This is the axial toroidal state in which time-reversal symmetry is preserved. Note that $\boldsymbol{\Omega}_{\boldsymbol{A}} \propto \sum_{i} \boldsymbol{r}_{i} \times \boldsymbol{p}_{i}$ is the axial toroidal moment. (b) Magneto-toroidal state with a vortexlike arrangement of spin $s$. This is a polar toroidal state in which timereversal symmetry is broken. Note that $\boldsymbol{\Omega}_{\boldsymbol{P}} \propto \sum_{i} \boldsymbol{r}_{i} \times \boldsymbol{s}_{i}$ is the polar toroidal moment. (c) Anapole state. The loop currents $j$ flowing on the surface of a torus induce toroidal magnetic fields $\boldsymbol{m}$. Note that $\boldsymbol{\Omega}_{\boldsymbol{P}} \propto \int \boldsymbol{r} \times \boldsymbol{m}(\boldsymbol{r}) d^{3} r$ is the anapole vector.

plane, transnational symmetry is not broken, but spatial inversion and fourfold rotational symmetries are broken. The anapole state has been discussed in the pseudogap state in cuprates [12-21], but the presence of such a state has been highly controversial [22-24].

Recently, the layered perovskite $\mathrm{Sr}_{2} \mathrm{IrO}_{4}$ has attracted much interest because it is the first experimental realization of a spin-orbit coupled Mott insulator [25-29]. The combination of strong spin-orbit coupling and electron correlation makes this material a promising candidate that hosts a novel electronic state of matter. The crystal has a tetragonal structure of corner sharing $\mathrm{IrO}_{6}$ octahedra, which is rotated by $\theta=11^{\circ}$ around the $c$ axis [Figs. 2(a) and 2(b)] [30-33]. A crystalline electric field splits the energy levels of $5 d^{5}$ (a)

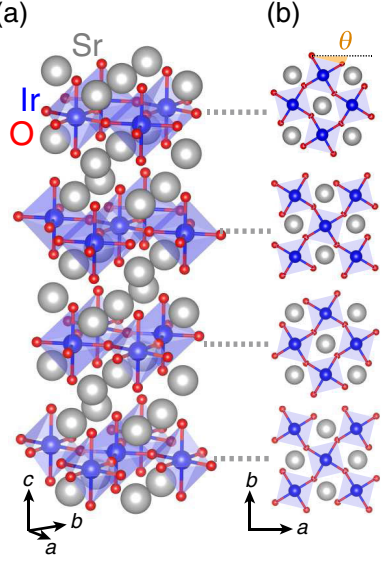

(c)

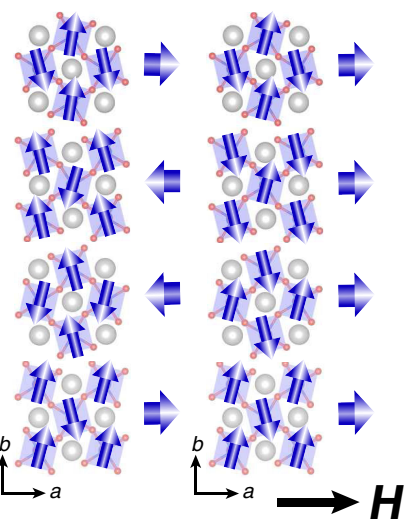

FIG. 2. (a) Crystal structure of $\mathrm{Sr}_{2} \mathrm{IrO}_{4}$. (b) Top view of each layer. The crystal has a tetragonal structure with corner sharing $\mathrm{IrO}_{6}$ octahedra, which is rotated by $\theta=11^{\circ}$ around the $c$ axis. (c) AF-I (+--+) magnetic structure. (d) AF-II $(++++)$ magnetic structure. Weak ferromagnetic moments are induced in the plane. electrons in the $\mathrm{Ir}^{4+}$ ion into $e_{g}$ and $t_{2 g}$ states. The presence of a strong spin-orbit interaction splits $t_{2 g}$ states into a pseudospin $J_{\text {eff }}=1 / 2$ doublet and $J_{\text {eff }}=3 / 2$ quartet. A large enough Coulomb interaction splits the $J_{\text {eff }}=1 / 2$ doublet, leading to a Mott insulating state with one localized electron, which is well described by the $J_{\text {eff }}=1 / 2$ pseudospin anisotropic Heisenberg model [25]. Such a state has been experimentally established by angle-resolved photoemission spectroscopy (ARPES) and resonant x-ray scattering experiments [25,34]. Below $T_{N} \approx 240 \mathrm{~K}, \mathrm{Sr}_{2} \mathrm{IrO}_{4}$ exhibits a long-range antiferromagnetic (AFM) order [31,32,34]. The magnetic moments are aligned in the basal $a b$ plane, and they follow the rotation of $\mathrm{IrO}_{6}$ octahedra forming a canted antiferromagnetism with $(+--+)$ structure along the $c$ axis (AF-I), as illustrated in Fig. 2(c) [34,35]. When a magnetic field of greater than or about $0.1 \mathrm{~T}$ is applied parallel to the $a b$ plane, the magnetic structure of AF-I changes to that of AF-II with $(++++)$ structure as shown in Fig. 2(d), in which weak ferromagnetic moments are induced within the plane [34].

Despite the complication of the strong spin-orbit interaction, different active orbitals, and structural distortions, it has been suggested that the low-energy electronic structure of $\mathrm{Sr}_{2} \mathrm{IrO}_{4}$ bears a certain resemblance to that of the cuprates $[25,36]$. It has been pointed out that electrondoped iridates should be compared to hole-doped cuprates because of the opposite band curvature due to the opposite sign of the next-nearest hopping term [37]. In electrondoped $\mathrm{Sr}_{2} \mathrm{IrO}_{4}$, which is achieved by $\mathrm{K}$-doping at the surface or by partial substitution of $\mathrm{La}$ for $\mathrm{Sr}$ in the bulk [38,39], ARPES and scanning tunneling microscopy measurements report the emergence of Fermi surface pockets, Fermi arcs, and $d$-wave-like pseudogaps [40-45], but no direct signature of the superconductivity has been reported, despite the theoretical predictions [37,46-48].

The effective hole doping is achieved by $\mathrm{Rh}$ substitution $[49,50]$. It has been shown that $\mathrm{Rh}$ ions act as acceptors by forming a $\mathrm{Rh}^{3+}\left(4 d^{6}\right)$ oxidation state, creating nearby $\operatorname{Ir}^{5+}\left(5 d^{4}\right)$ ions to preserve the charge neutrality [51]. The $T-x$ phase diagram of $\operatorname{Sr}_{2}\left(\operatorname{Ir}_{1-x} \mathrm{Rh}_{x}\right) \mathrm{O}_{4}$ is displayed in Fig. 3. The AFM transition temperature decreases almost linearly as a function of $x$ and vanishes at a critical doping of $x_{c} \sim 0.17$ [51]. The AF-I-type magnetic structure is preserved in the low- $x$ regime. At $x \gtrsim 0.04-0.06$, a long-range order (LRO) phase with AF-I type structure is realized below $T_{N}^{L}$, while a short-range order (SRO) phase appears between $T_{N}^{S}$ and $T_{N}^{L}$ [51]. The ARPES measurements report that the $J_{\text {eff }}=1 / 2$ band reaches the Fermi level, forming small hole pockets around the $X$ point at $x \sim 0.07-0.10$ [52,53]. The pseudogaplike behavior is also reported in the hole-doped $\mathrm{Sr}_{2} \mathrm{IrO}_{4}$ by ARPES [52,53], although there are several distinct differences in the low-energy electronic structure between $\mathrm{Sr}_{2}\left(\mathrm{Ir}_{1-x} \mathrm{Rh}_{x}\right) \mathrm{O}_{4}$ and electron-doped cuprates.

The phase diagram of cuprates features a plethora of broken symmetries in the pseudogap regime, which 


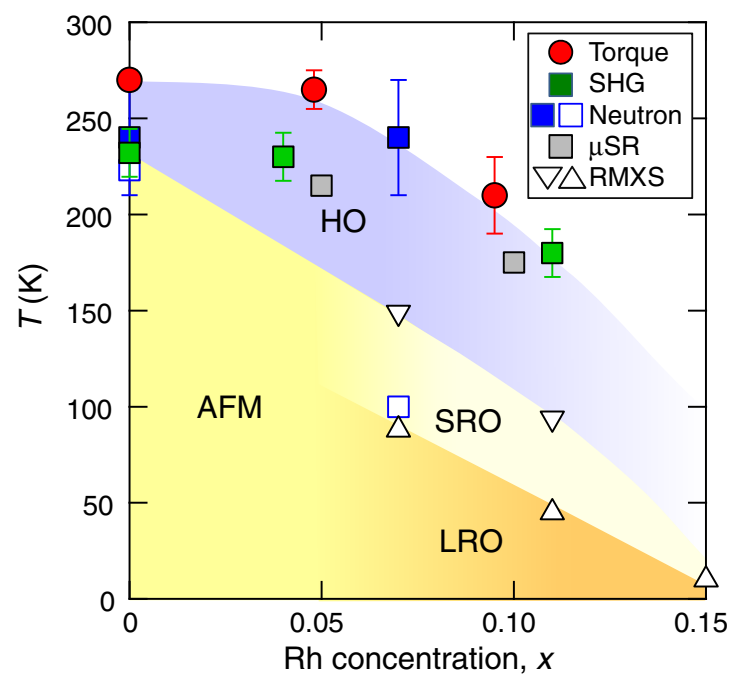

FIG. 3. $T-x$ phase diagram of $\mathrm{Sr}_{2}\left(\operatorname{Ir}_{1-x} \mathrm{Rh}_{x}\right) \mathrm{O}_{4}$ determined by the SHG, polarized neutron, resonant magnetic x-ray scattering (RMXS) muon spin relaxation, and the present torque measurements. The blue region represents the hidden-order $(\mathrm{HO})$ phase. In the AFM phase, the AF-I magnetic structure at low fields changes to the AF-II structure in parallel fields above about $0.1 \mathrm{~T}$. At $x \gtrsim 0.06$, the magnetic short-range order (SRO) occurs at high temperature, which is followed by magnetic long-range order (LRO) when the AF-II structure occurs.

includes charge-density-wave, stripe, nematic, and possible loop-current orders [54]. Therefore, it is highly intriguing to investigate the symmetry-breaking phase proximate to the spin-orbit coupled Mott insulator $\mathrm{Sr}_{2} \mathrm{IrO}_{4}$. Recently, the presence of a hidden broken symmetry phase that develops prior to the AFM phase has been reported in pure and $\mathrm{Rh}$ substituted $\mathrm{Sr}_{2} \mathrm{IrO}_{4}$ by optical second-harmonic generation (SHG) measurements [55]. The SHG can sensitively detect odd-parity order parameters but is insensitive to even-parity order parameters [55-57]. In Fig. 3, the onset temperature of the hidden order determined by the SHG signal $T_{\Omega}$ is plotted. For pure $\mathrm{Sr}_{2} \mathrm{IrO}_{4}, T_{\Omega}$ appears to be a few Kelvin above $T_{N}$, and for Rh-doped $\mathrm{Sr}_{2} \mathrm{IrO}_{4}, T_{\Omega}$ is distinctly far above $T_{N}$. These results suggest that an odd-parity hiddenorder phase develops at higher temperatures above the AFM transition.

Subsequent polarized neutron diffraction measurements in pure and 7\% Rh-doped $\mathrm{Sr}_{2} \mathrm{IrO}_{4}$ report the development of an unconventional magnetic order that breaks time-reversal symmetry above about $T_{N}$, which is characterized by an intra-unit-cell magnetic order [58]. The onset temperature of this order matches $T_{\Omega}$ determined by SHG. It should be noted that a similar unconventional magnetic order has been reported in the pseudogap state of underdoped cuprates, including $\mathrm{YBa}_{2} \mathrm{Cu}_{3} \mathrm{O}_{6+\delta}$ [18], $\mathrm{HgBa}_{2} \mathrm{CuO}_{4+\delta}$ [19], and $\mathrm{Bi}_{2} \mathrm{Sr}_{2} \mathrm{CaCu}_{2} \mathrm{O}_{y}$ [20]. The intraunit-cell magnetism has been discussed in terms of possible counter-circulating loop currents within the unit cell of $\mathrm{IrO}_{2}$ or $\mathrm{CuO}_{2}$ planes (Varma's loop) [12-14]. Very recent muon spin relaxation $(\mu \mathrm{SR})$ measurements on $\mathrm{Sr}_{2}\left(\mathrm{Ir}_{1-x} \mathrm{Rh}_{x}\right) \mathrm{O}_{4}$, with $x=0.05$ and 0.1 , report the critical slowing down of electronic spin fluctuations at about $T_{\Omega}$ [59]. Similar phenomena have been reported in the pseudogap state of cuprates [60]. It has been claimed that these muon results are consistent with the polarized neutron experiments. The onset temperatures of the unconventional magnetic order reported by neutron and $\mu \mathrm{SR}$ measurements are plotted in Fig. 3. Since there is no evidence of structural distortions that break translational symmetry above $T_{N}$, the hiddenorder state is suggested to be a possible anapole state.

Despite the SHG, polarized neutron diffraction, and $\mu \mathrm{SR}$ measurements, however, the nature of the hidden-order state remains largely elusive. To study this state, it is crucially important to clarify the spatial symmetry breaking by a thermodynamic bulk probe. Moreover, although polarized neutron measurements suggest that the direction of the magnetic moment is perpendicular to the $a b$ plane, the direction of the anapole vector is unknown [58]. As there are several patterns of the loop current [12-17], determining the direction of the anapole vector is essentially important for understanding the origin of the broken time-reversal symmetry. In Figs. 4(a) and 4(b), two examples of the anapole vector and loop-current patterns are illustrated.

In this paper, to obtain deep insight into the symmetrybreaking phenomena in the hidden-order phase of $\mathrm{Sr}_{2}\left(\mathrm{Ir}_{1-x} \mathrm{Rh}_{x}\right) \mathrm{O}_{4}$, we measure the magnetic susceptibility anisotropy within the $\mathrm{IrO}_{2}$ plane with exceptionally high-precision magnetic torque experiments [61-64] and (a)
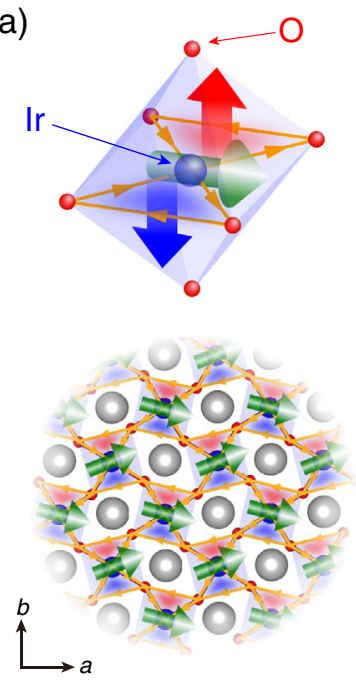

(b)

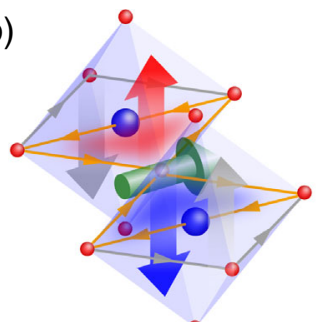

0

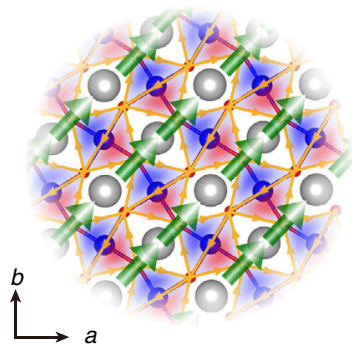

FIG. 4. (a) Upper figure: loop currents (thin orange arrows), induced magnetic fields (thick red and blue arrows), and anapole vector (green arrow) in the $\mathrm{IrO}_{6}$ octahedron. The net anapole vector is along the [100] Ir-Ir direction. The lower figure is the top view of the upper figure. (b) Same as (a), but the loop current pattern is different. The anapole vector is along the [110] Ir-O-Ir bond direction. 
nematic susceptibility with elastoresistance [65-70]. We find that a distinct $C_{2}$ in-plane anisotropy that breaks $C_{4}$ symmetry of the underlying lattice sets in below about $T_{\Omega}$ in $\operatorname{Sr}_{2}\left(\operatorname{Ir}_{1-x} \mathrm{Rh}_{x}\right) \mathrm{O}_{4}$. This finding provides thermodynamic evidence for a nematic transition, which bears resemblance to the phenomena observed in the pseudogap state of cuprates $[63,64]$. However, the nematic director of $\mathrm{Sr}_{2}\left(\mathrm{Ir}_{1-x} \mathrm{Rh}_{x}\right) \mathrm{O}_{4}$ is along the Ir-O-Ir bond direction, in stark contrast to that of the monolayer cuprate $\mathrm{HgBa}_{2} \mathrm{CuO}_{4+\delta}$, which is $45^{\circ}$ rotated from the $\mathrm{Cu}-\mathrm{O}-\mathrm{Cu}$ bond direction [64]. Moreover, in contrast to iron-based superconductors, in which the nematic susceptibility is largely enhanced towards the nematic transition [65-69], the nematic susceptibility of $\operatorname{Sr}_{2}\left(\operatorname{Ir}_{1-x} \mathrm{Rh}_{x}\right) \mathrm{O}_{4}$ exhibits no divergent behavior towards $T_{\Omega}$, which is consistent with the odd-parity order parameter. Based on these results, we discuss the hidden-order state in terms of an anapole state with peculiar loop-current patterns.

\section{EXPERIMENTAL}

Measurements of the magnetic torque $\boldsymbol{\tau}=\mu_{0} V \boldsymbol{M} \times \boldsymbol{H}$ have a high sensitivity for the detection of magnetic anisotropy, where $\mu_{0}$ is space permeability, $V$ is the sample volume, and $\boldsymbol{M}$ is the magnetization induced by external magnetic field $\boldsymbol{H}$. Torque is a thermodynamic quantity that is given by the derivative of the free energy with respect to angular displacement, and it can thus shed light on the thermodynamic character of the transition. We perform the torque measurements for a range of directions of $\boldsymbol{H}$ within the tetragonal $a b$ plane of $\mathrm{Sr}_{2}\left(\mathrm{Ir}_{1-x} \mathrm{Rh}_{x}\right) \mathrm{O}_{4}$ to test whether the hidden-order breaks fourfold crystal symmetry. In this configuration, $\tau$ is a periodic function of twice the azimuthal angle $\phi$ measured from the $a$ axis:

$$
\tau_{2 \phi}=\frac{1}{2} \mu_{0} V H^{2}\left[\left(\chi_{a a}-\chi_{b b}\right) \sin 2 \phi-2 \chi_{a b} \cos 2 \phi\right],
$$

where $\chi_{i j}$ is the susceptibility tensor defined as $M_{i j}=$ $\sum_{j} \chi_{i j} H_{j}(i, j=a, b, c)$. For a tetragonally symmetric system, $\tau_{2 \phi}$ should be zero because $\chi_{a a}=\chi_{b b}$ and $\chi_{a b}=0$. Nonzero values of $\tau_{2 \phi}$ appear when the tetragonal symmetry is broken by a new electronic or magnetic state; $C_{4}$ rotational symmetry breaking is revealed by $\chi_{a a} \neq \chi_{b b}$ and/or $\chi_{a b} \neq 0$. The former and the latter states are illustrated in Figs. 5(a) and 5(b), respectively, where $C_{4}$ symmetry breaking occurs along the $[100] /[010]$ direction ( $B_{1 g}$ symmetry) and the [110] direction (bond-directional nematicity with $B_{2 g}$ symmetry) of the $\mathrm{IrO}_{2}$ plane.

For the in-plane torque magnetometry, we use a highly sensitive piezoresistive cantilever [61-64]. The experimental setup for this measurement is illustrated in Fig. 6 (a). To measure the in-plane variation of the magnetic torque, we use a system with two superconducting
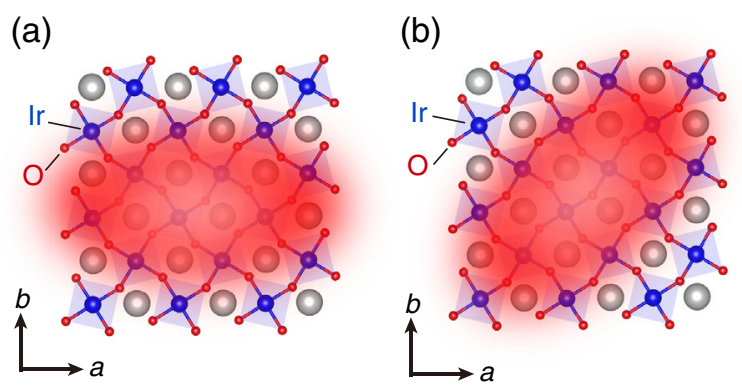

FIG. 5. (a) Schematic picture of the nematicity with $B_{1 q}$ symmetry, where the nematic director is along the Ir-Ir [100] direction. For this nematicity, $\chi_{a a} \neq \chi_{b b}$ and $\chi_{a b}=0$. (b) Nematicity with $B_{2 g}$ symmetry, where the nematic director is along the [110] Ir-O-Ir direction. For this nematicity, $\chi_{a a}=\chi_{b b}$ and $\chi_{a b} \neq 0$.

magnets generating magnetic fields in two mutually orthogonal directions and a cryostat set on a mechanical rotating stage at the top of a dewar [61-64]. By computercontrolling the two superconducting magnets and the rotating stage, $\boldsymbol{H}$ is precisely applied and rotated within the $a b$ plane. We carefully check the misalignment of $\boldsymbol{H}$ from the $a b$ plane by rotating $\boldsymbol{H}$ conically about the $c$ axis and find the misalignment is less than $0.1^{\circ}$. To obtain the maximum torque signal, we apply $\mu_{0}|\boldsymbol{H}|=4 \mathrm{~T}$, which is the highest field available in our vector magnet system. When the nematicity appears in the tetragonal lattice, the formation of nematic domains is naturally expected. In the presence of a large number of domains, the twofold oscillations due to nematicity are canceled out. According to the SHG measurements, the typical domain size is 50-100 $\mu \mathrm{m}$ [55]. Therefore, we use crystals with sizes of less than or about $100 \mu \mathrm{m}$ in the torque measurements. Since such crystals contain a very small number of domains, the twofold oscillations can be detected if nematicity appears.
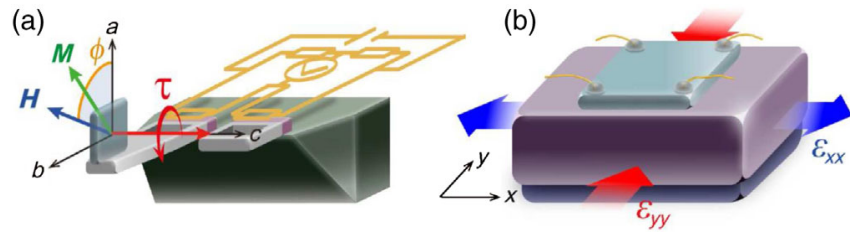

FIG. 6. (a) Experimental setup of in-plane magnetic torque measurements. Magnetic torque $\boldsymbol{\tau}=\mu_{0} \boldsymbol{V H} \times \boldsymbol{M}$ is detected by a microcantilever. Here, $\boldsymbol{H}$ is rotated within the $a b$ plane. (b) Experimental setup of elastoresistance measurements. The sample (light blue) is glued on the piezo stack (purple), and the resistances $R_{x x}$ and $R_{y y}$ along the $x$ and $y$ directions, respectively, are measured by the van der Pauw method. The strain $\varepsilon_{x x}$ along $x$ is measured by a strain gauge attached on the back side of the piezo stack. The strain $\varepsilon_{y y}$ along $y$ is calculated by the temperature-dependent Poisson ratio, which has been calibrated in advance. 
The nematic susceptibility is determined by measuring the elastoresistance. The experimental setup for this measurement is illustrated in Fig. 6(b). Two samples are cut from a single crystal of $\mathrm{Sr}_{2} \mathrm{Ir}_{0.88} \mathrm{Rh}_{0.12} \mathrm{O}_{4}$, and the crystal axes of the samples are determined by $\mathrm{x}$-ray diffraction. Four contacts are attached near the corners of the samples by silver paste and cured at about $250^{\circ} \mathrm{C}$. The two samples are glued on the piezo stack, whose $x$ direction is aligned along the [100] and [110] directions, respectively. The van der Pauw method is used to measure resistance changes $\Delta R_{x x}$ and $\Delta R_{y y}$ along the $x$ and $y$ directions of the piezo stack $[67,69]$. The difference $\eta=$ $(\Delta R / R)_{x x}-(\Delta R / R)_{y y}$ is the anisotropy induced by the anisotropic strain $\varepsilon_{x x}-\varepsilon_{y y}$, which is measured by the strain gauge. The quantity $\eta$ is expected to be intimately linked to an electronic nematic-order parameter. When the anisotropic strain couples linearly to the nematic order, the nematic susceptibility can be defined as the quantity

$$
\chi_{\mathrm{nem}} \equiv d\left[(\Delta R / R)_{x x}-(\Delta R / R)_{y y}\right] / d\left(\varepsilon_{x x}-\varepsilon_{y y}\right) .
$$

The measurements of two directions along [100] and [110] correspond to two irreducible representations ( $B_{1 g}$ and $B_{2 g}$ ) for the tetragonal $D_{4 h}$ system.

$$
x=0
$$

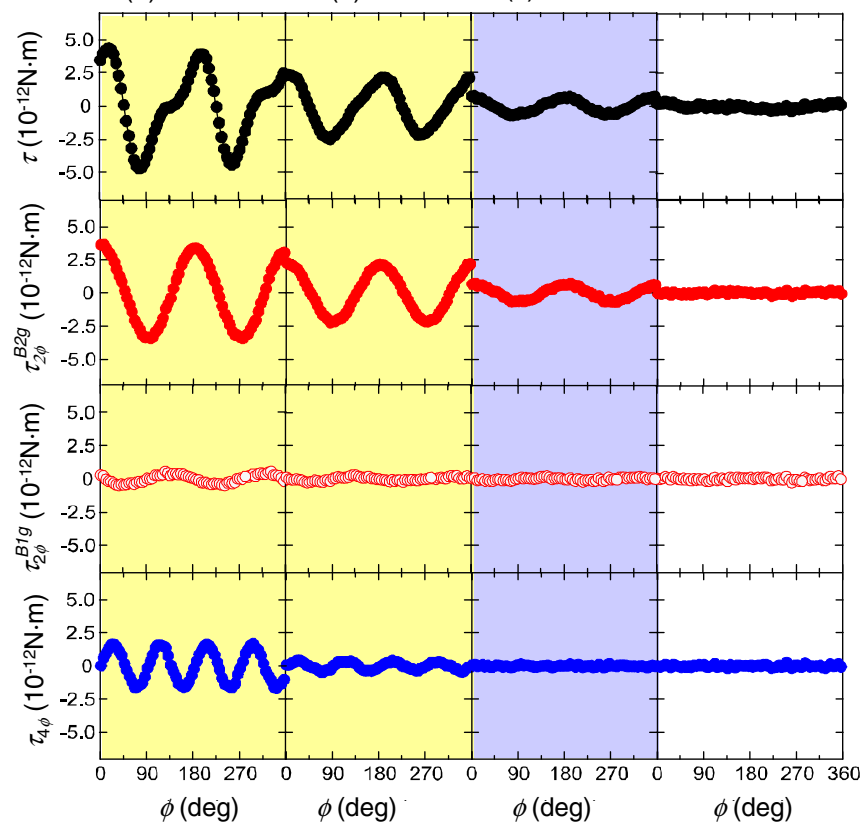

\section{RESULTS}

\section{A. Magnetic torque}

Top panels of Figs. 7(a)-7(d) and 7(e)-7(h) display the raw data of the magnetic torque $\tau(\phi)$ in the magnetic field of $\mu_{0}|\boldsymbol{H}|=4 \mathrm{~T}$ rotating within the $a b$ plane at several temperatures for pure $\mathrm{Sr}_{2} \mathrm{IrO}_{4}$ and $\mathrm{Sr}_{2} \mathrm{Ir}_{0.905} \mathrm{Rh}_{0.095} \mathrm{O}_{4}$ $\left(T_{N}^{S} \approx 120 \mathrm{~K}, T_{N}^{L} \approx 80 \mathrm{~K}\right)$, respectively. Note that we use the unit cell with the space group $I 4_{1} / a$, where the $a$ axis corresponds to the Ir-Ir direction, as shown in Figs. 2(a) and 2(b). This unit cell is $45^{\circ}$ rotated from the unit cell of cuprates, where the $a$ axis corresponds to the $\mathrm{Cu}-\mathrm{O}-\mathrm{Cu}$ bond direction. All torque curves are perfectly reversible with respect to the field rotation direction, indicating no detectable ferromagnetic impurities. For both crystals, no oscillations are observed in $\tau(\phi)$ at the highest temperatures, which is consistent with the tetragonal crystal symmetry. At lower temperatures, $\tau(\phi)$ exhibits distinct angular dependence. We analyze $\tau(\phi)$ by decomposing as $\tau=\tau_{2 \phi}+\tau_{4 \phi}$, where $\tau_{n \phi}=A_{n \phi} \sin \left[n\left(\phi-\phi_{n 0}\right)\right]$ is a term with $n$-fold symmetry. The twofold component is further decomposed as $\tau_{2 \phi}=A_{2 \phi}^{B 1 g} \sin 2 \phi+A_{2 \phi}^{B 2 g} \cos 2 \phi$. In the upper-middle, lower-middle, and bottom panels of Figs. 7(a)-7(h), the $B_{2 g}$ twofold, $B_{1 g}$ twofold, and fourfold components obtained from the Fourier analysis of the raw

$$
x=0.095
$$

(e) $60 \mathrm{~K}$

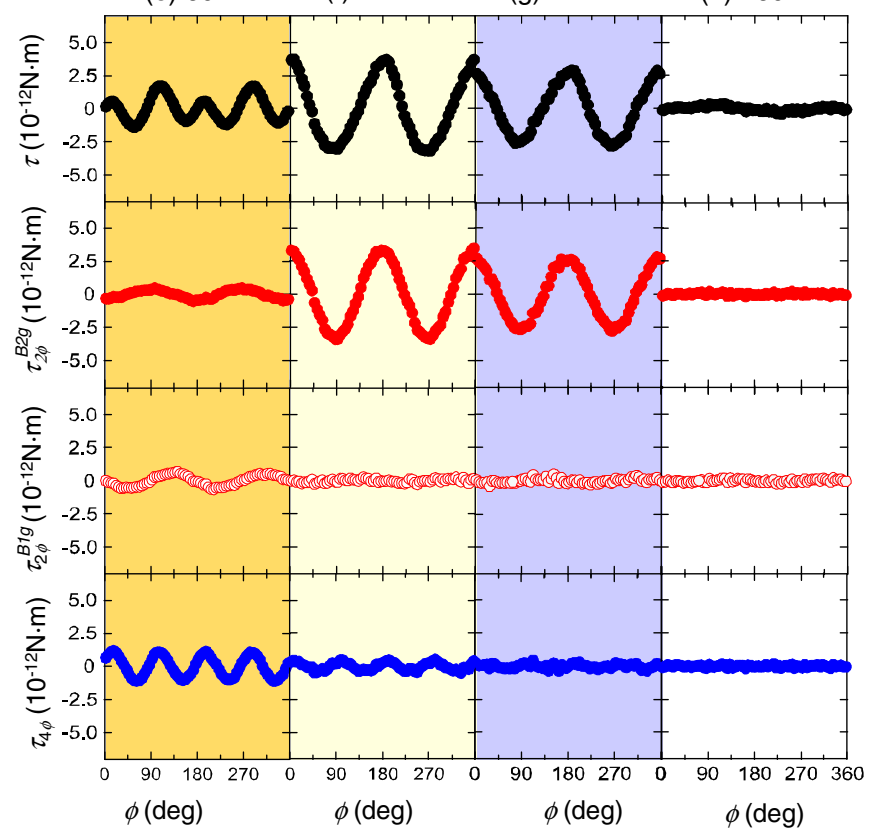

FIG. 7. Rows (a)-(d) and (e)-(h) display the magnetic torque $\tau(\phi)$ in the in-plane magnetic field of $\left|\mu_{0} \boldsymbol{H}\right|=4 \mathrm{~T}$ as a function of the azimuthal angle $\phi$ for pure $\mathrm{Sr}_{2} \mathrm{IrO}_{4}\left(T_{N} \approx 240 \mathrm{~K}\right)$ and $\mathrm{Sr}_{2} \mathrm{Ir}_{0.905} \mathrm{Rh}_{0.095} \mathrm{O}_{4}\left(T_{N}^{S} \approx 120 \mathrm{~K}\right.$ and $\left.T_{N}^{L} \approx 80 \mathrm{~K}\right)$, respectively. For $x=0$, white, blue, and yellow shaded regions show that the system is in the paramagnetic, hidden-order, and AFM states, respectively. For $x=0.095$, the orange (light-yellow) region shows that the system is in the long-range (short-range) AFM state. The top panels show raw torque curves $\tau(\phi)$. The upper-middle, lower-middle, and bottom panels show the $B_{2 g}$ twofold ( $\cos 2 \phi$ ), $B_{1 g}$ twofold (sin $2 \phi$ ), and fourfold $(\sin 4 \phi)$ components of the torque curves, respectively, obtained from Fourier analysis of the raw torque curves. 
torque curves are displayed. The raw $\tau(\phi)$ curves are well reproduced by the sum of these three components.

As shown in the upper middle panels of Figs. 7(a)-7(g), distinct twofold oscillation with $B_{2 g}$ symmetry $(\propto \cos 2 \phi)$ is observed at $270 \mathrm{~K}$ for $\mathrm{Sr}_{2} \mathrm{IrO}_{4}$ and at $140 \mathrm{~K}$ for $\mathrm{Sr}_{2} \mathrm{Ir}_{0.905} \mathrm{Rh}_{0.095} \mathrm{O}_{4}$. It should be stressed that these temperatures are obviously higher than $T_{N}$. Therefore, the data provide the first evidence for a phase transition to an electronic nematic state as a bulk property. As shown in the lower-middle and bottom panels of Figs. 7(a)-7(h), small but finite twofold oscillations with $B_{1 g}$ symmetry $(\propto \sin 2 \phi)$ and fourfold oscillations, which have the form $\tau_{4 \phi}=A_{4 \phi} \sin 4 \phi$, are observed at low temperatures.

Figures 8(a)-8(c) depict the temperature dependence of the amplitude of each oscillation for $\mathrm{Sr}_{2}\left(\mathrm{Ir}_{1-x} \mathrm{Rh}_{x}\right) \mathrm{O}_{4}$, with $x=0$ (pure), 0.048, and 0.095, respectively. In all crystals, finite twofold $B_{2 g}$ oscillations appear well above $T_{N}$ or $T_{N}^{S}$. Moreover, the amplitude of twofold oscillations grows nearly linearly with decreasing temperature as $A_{2 \phi}^{B 2 g} \propto\left|T_{\Omega}-T\right|^{\beta}$, with $\beta \approx 1$. The onset temperature of the twofold $B_{2 g}$ oscillations is plotted in the $T-x$ phase diagram displayed in Fig. 3. We note that the onset temperature of the twofold $B_{2 g}$ oscillations does not depend on the magnetic field strength. For pure $\mathrm{Sr}_{2} \mathrm{IrO}_{4}$, the onset temperature of twofold $B_{2 q}$ oscillations is slightly higher than the onset temperatures reported by the SHG and polarized neutron scattering measurements $[55,58]$. In Rhdoped $\mathrm{Sr}_{2} \mathrm{IrO}_{4}$, on the other hand, the onset temperature appears to be close to that determined by the SHG and neutron measurements. Considering the ambiguity in determining the onset temperature of the SHG signal and the neutron diffraction intensity, the onset temperature of the tetragonal $C_{4}$ symmetry breaking appears to match that of the hidden-order $T_{\Omega}$. Thus, the present results provide thermodynamic evidence of the nematic transition at about $T_{\Omega}$.

For pure $\mathrm{Sr}_{2} \mathrm{IrO}_{4}$ and $x=0.048, A_{2 \phi}^{B 2 g}(T)$ increases with decreasing temperature with no discernible change at $T_{N}$
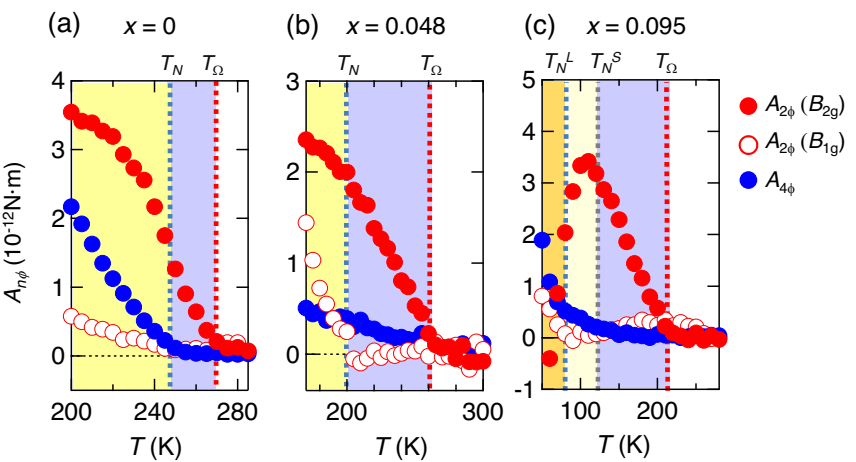

FIG. 8. Temperature dependence of the amplitude of twofold (red filled and open circles) and fourfold oscillations (filled blue circles) for pure and Rh-doped $\mathrm{Sr}_{2} \mathrm{IrO}_{4}$. (a) $x=0$, (b) $x=0.048$, and (c) $x=0.095$. and shows a saturating behavior at lower temperatures. These results suggest that the nematicity is insensitive to the long-range AFM order. In contrast, for $0.095, A_{2 \phi}^{B 2 g}(T)$ is strongly suppressed below about $T_{N}^{S}$ and changes sign below $T_{N}^{L}$, suggesting that the $B_{2 g}$ nematicity is strongly influenced by the appearance of short-range magnetic structure. With decreasing temperature, the amplitude of fourfold oscillations increases below $T_{N}$ for pure $\mathrm{Sr}_{2} \mathrm{IrO}_{4}$ $[71,72]$. The torque curves are perfectly reversible with respect to the field rotation below $T_{N}$, indicating that the induced weak ferromagnetic moments completely follow the applied $\boldsymbol{H}$ rotated within the $a b$ plane. Moreover, the fourfold oscillations for $x=0.048$ and 0.095 appear even above the AFM-ordering temperatures. These results indicate that the fourfold oscillations are not caused by the weak ferromagnetic moments shown in Fig. 2(d) but arise primarily from the nonlinear susceptibilities [61].

\section{B. Elastoresistance}

The elastoresistance measurements have been used as a powerful probe of electronic nematic transitions in strongly correlated electron systems, especially in iron-based superconductors $[65,66,68,69,73]$. When the nematic order couples linearly to the strain, this technique can quantify the nematic susceptibility, which is related to the fluctuations of a rotational-symmetry-breaking order above the nematic transition. Thus, this method is complementary to the magnetic torque measurements, which measure the nematic-order parameter below the transition.

The results of elastoresistance measurements along two different strain directions are shown in Figs. 9(a)-(d). The linearity of the resistance change with respect to the strain observed for both $x$ and $y$ directions indicates that the anisotropy $\eta$ is proportional to the strain. The doping level of the samples is $x=0.12$, where the long-range antiferromagnetic and hidden-order transition temperatures are expected to be about $30 \mathrm{~K}$ and $150 \mathrm{~K}$ (see Fig. 3). As shown in Fig. 10(a), the temperature dependence of the nematic susceptibility $\chi_{\text {nem }}=d\left[(\Delta R / R)_{x x}-(\Delta R / R)_{y y}\right] / d\left(\varepsilon_{x x}-\varepsilon_{y y}\right)$ along [100] shows a gradual increase with decreasing temperature, but it does not show any divergent behavior near the putative hidden-order transition around $150 \mathrm{~K}$. The data for the [110] direction [Fig. 10(b)], which is along the nematic director expected from the torque measurements, show even weaker temperature dependence with no discernible anomaly around $150 \mathrm{~K}$. In both directions, an enhancement is observed below $30 \mathrm{~K}$ below the Néel temperature $T_{N}^{L}$, where the ground state is insulating. The observed absence of the divergent behavior in two directions, which correspond to two irreducible representations $B_{1 g}$ and $B_{2 g}$ in the tetragonal $D_{4 h}$ system, provides evidence against even-parity nematic order in the hidden-order phase of $\mathrm{Sr}_{2} \mathrm{Ir}_{1-x} \mathrm{Rh}_{x} \mathrm{O}_{4}$. Therefore, this evidence implies that the rotational symmetry-breaking 

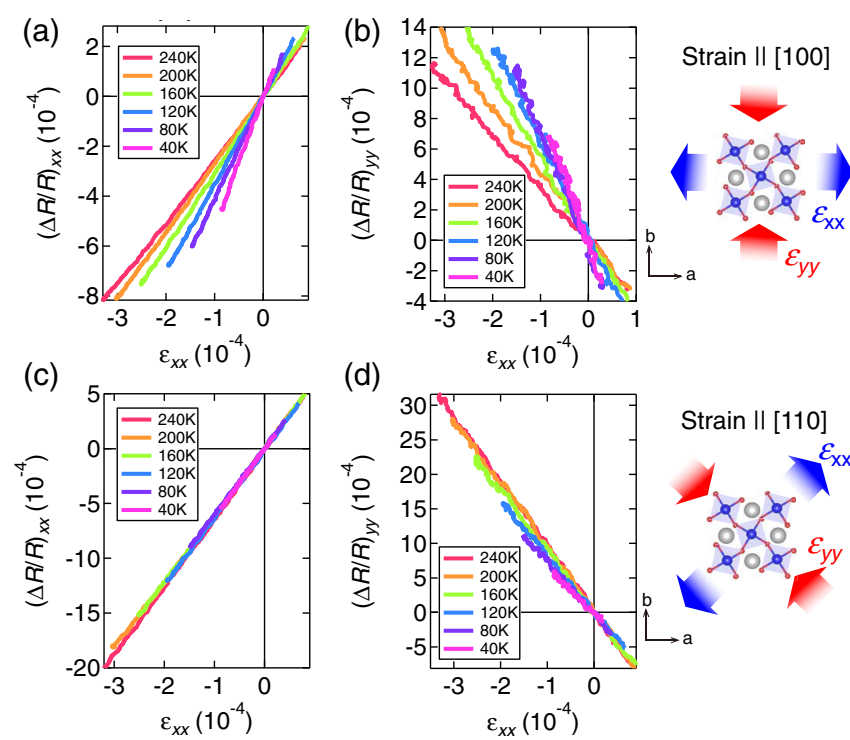

(d)

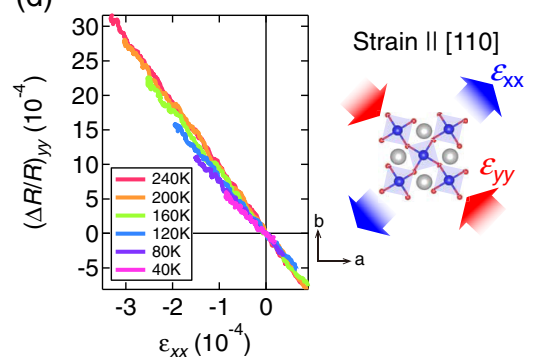

FIG. 9. Representative data of elastoresistance for $\mathrm{Sr}_{2}\left(\mathrm{Ir}_{1-x} \mathrm{Rh}_{x}\right) \mathrm{O}_{4}$ with $x=0.12$. (a) Longitudinal elastoresistance $(\Delta R / R)_{x x}$ and (b) transverse elastoresistance $(\Delta R / R)_{y y}$ for several temperatures as a function of strain $\varepsilon_{x x}$ with the strain direction $x$ parallel to [100]. (c) Longitudinal elastoresistance $(\Delta R / R)_{x x}$ and (d) transverse elastoresistance $(\Delta R / R)_{y y}$ for several temperatures as a function of strain $\varepsilon_{x x}$ with the strain direction $x$ parallel to [110]. The linear response of the resistance against the strain can be seen in all the elastoresistance data.

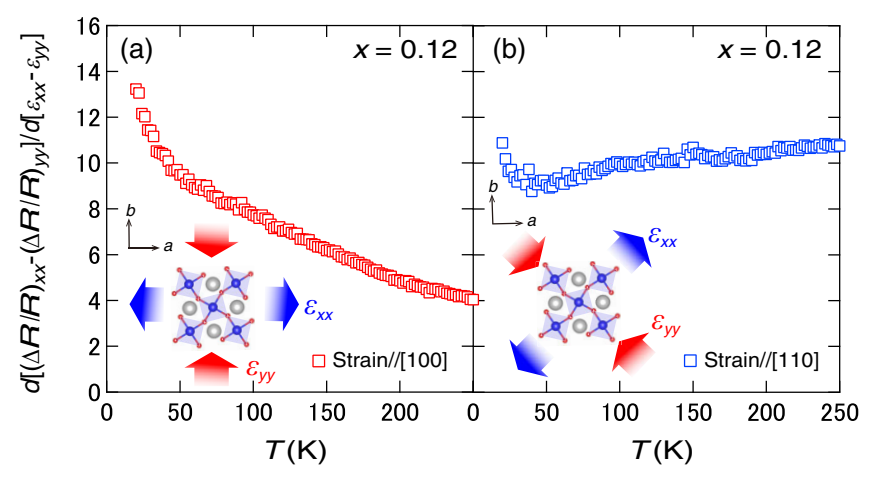

FIG. 10. Temperature dependence of nematic susceptibility in $\mathrm{Sr}_{2} \mathrm{Ir}_{0.88} \mathrm{Rh}_{0.12} \mathrm{O}_{4}$. (a) Strain applied along the [100] direction. (b) Strain applied along the [110] direction. The insets illustrate the directions of applied strain and the $\mathrm{IrO}_{6}$ octahedra.

state revealed by the magnetic torque is of odd parity, as will be discussed later.

\section{DISCUSSION}

Our main experimental findings for pure and $\mathrm{Rh}$ substituted $\mathrm{Sr}_{2} \mathrm{IrO}_{4}$ are threefold: First, the in-plane torque magnetometry provides thermodynamic evidence of the nematic transition that lowers the rotational symmetry of the system from $C_{4}$ to $C_{2}$ at about $T_{\Omega}$. Second, the growth of $B_{2 g}$ twofold oscillations indicates a nematic director along the Ir-O-Ir [110] bond direction, in contrast to previous proposals [55,58]. Third, the elastoresistance shows no diverging nematic susceptibility towards $T_{\Omega}$. We note that the possibility of the field-induced twofold oscillations in the paramagnetic phase can be excluded because of the following reason: When the in-plane anisotropy is induced by the external magnetic field, the direction of the anisotropy is expected to follow the rotation of the magnetic field. Therefore, the fieldinduced anisotropy, even if present, does not induce the twofold oscillations in the paramagnetic phase above $T_{N}$. Moreover, we point out that these results are consistent with the anapole state, which has been proposed by SHG and polarized neutron measurements [55,58]. It should be noted that the anapole-order parameter breaks inversion symmetry while the magnetic field preserves it. Thus, the coupling between the anapole moment and external magnetic field is not linear but arises from a second-order effect. This coupling gives rise to twofold magnetic anisotropy, which is detected as a twofold oscillation of the in-plane magnetic torque. Moreover, below $T_{\Omega}$, the amplitude of the twofold oscillations increases nearly linearly, which implies that the nematicity is not a primary order parameter but a secondary one of the hidden-order state.

In Fe-based superconductors, including $\mathrm{Ba}\left(\mathrm{Fe}_{1-x} \mathrm{Co}_{x}\right)_{2} \mathrm{As}_{2}, \mathrm{BaFe}_{2}\left(\mathrm{As}_{1-x} \mathrm{P}_{x}\right)_{2}$, and $\mathrm{FeSe}_{1-x} \mathrm{~S}_{x}$, the nematic susceptibility exhibits diverging behavior toward the nematic transition temperature $[65,66,68]$. In addition, the divergent nematic susceptibility has also been reported in a cuprate $(\mathrm{Bi}, \mathrm{Pb})_{2} \mathrm{Sr}_{2} \mathrm{CaCu}_{2} \mathrm{O}_{8+\delta}$ near the pseudogap critical point [70]. Therefore, the absence of the divergent nematic susceptibility with approaching $T_{\Omega}$, despite the presence of a thermodynamic nematic transition, is in sharp contrast to the nematic transition reported in other strongly correlated electron systems. One may suspect that electrons couple with the lattice very weakly in $\mathrm{Sr}_{2}\left(\mathrm{Ir}_{1-x} \mathrm{Rh}_{x}\right) \mathrm{O}_{4}$. However, such a possibility is unlikely because the inherently strong spin-orbit interaction in $\mathrm{Sr}_{2}\left(\mathrm{Ir}_{1-x} \mathrm{Rh}_{x}\right) \mathrm{O}_{4}$ is expected to give rise to a strong coupling between the electrons and the lattice. The present results of $\mathrm{Sr}_{2}\left(\mathrm{Ir}_{1-x} \mathrm{Rh}_{x}\right) \mathrm{O}_{4}$ suggest that the hidden-order state is not a simple ferroic nematic-ordered phase characterized by the wave vector $\boldsymbol{q}=0$.

We stress that the absence of divergent nematic susceptibility is consistent with the odd-parity order for the following reasons. In the case of even-parity ferroic nematic order, the elastoresistance measurements can sensitively detect the divergent behavior of nematic susceptibility toward the transition temperature. If the order is of odd parity, on the other hand, the divergence may not be observed from the elastoresistance because the linear coupling between the strain and the odd-parity order parameter is prohibited. In fact, in the Landau theory, coupling of anapole order and strain is described as 
$F=F_{\text {lat }}+F_{\text {ana }}+\gamma_{1} T_{x} T_{y} \varepsilon_{x y}+\gamma_{2}\left(T_{x}^{2}-T_{y}^{2}\right)\left(\varepsilon_{x x}-\varepsilon_{y y}\right)$,

where $\boldsymbol{T}=\left(T_{x}, T_{y}\right)$ is a polar-vector order parameter for the anapole order in the $E_{u}$ representation, $\varepsilon_{i j}$ are strain, $\gamma_{1}$ and $\gamma_{2}$ are coupling constants, and $F_{\text {lat }}$ and $F_{\text {ana }}$ are the free energy of the lattice strain and anapole order, respectively. Any linear coupling of the anapole-order parameter and strain, $T_{i} \varepsilon_{j k}$, is prohibited because $T_{i}$ and $\varepsilon_{j k}$ have different spacial inversion and time-reversal symmetries; anapole order is odd parity while the strain is even parity. The absence of the linear couplings reveals no divergent susceptibility when approaching the anapole transition $T_{\Omega}$ from high temperatures, which is in contrast to the even-parity nematic case whose order parameters couple linearly to the strain. On the other hand, the bond-directional anapole-order parameter grows below $T_{\Omega}$ as $\left|T_{x}\right|=$ $\left|T_{y}\right| \propto\left(T_{\Omega}-T\right)^{1 / 2}$ in the mean field region. Through the coupling term in Landau free energy, nematicity $\varepsilon_{x y} \propto$ $T_{\Omega}-T$ is induced below $T_{\Omega}$. These expected behaviors in the mean field region of anapole order are compatible with the results of magnetic torque and elastoresistance measurements.

We now discuss the direction of the anapole moment and loop-current pattern in the hidden-order state. The polar toroidal moment that points to the $a$ axis and the simplest loop-current pattern illustrated in Fig. 4(a) have been proposed in the previous SHG and polarized neutron diffraction studies [55,58]. In our torque magnetometry, however, the twofold oscillations below $T_{\Omega}$ follow the functional form $\tau_{2 \phi}=A_{2 \phi} \cos 2 \phi$. This result clearly indicates $\chi_{a a}=\chi_{b b}$ and $\chi_{a b} \neq 0$ in Eq. (1), i.e., the emergence of the nematicity whose anisotropy axis is along the Ir-O-Ir direction, which is referred to as bond nematicity. The direction of the nematic director is the same as that of the anapole moment. Therefore, the torque magnetometry reveals that the anapole moment points to the [110] direction and rotated $45^{\circ}$ from the $a$ axis, as illustrated in Fig. 4(b).

Among the resemblances of crystallographic, magnetic, and electronic structures between $\mathrm{Sr}_{2} \mathrm{IrO}_{4}$ and $\mathrm{La}_{2} \mathrm{CuO}_{4}$, the formation of a pseudogap with the Fermi arc in electron-doped $\mathrm{Sr}_{2} \mathrm{IrO}_{4}$ has drawn a great deal of attention because the pseudogap is one of the most prominent and most discussed features of the cuprates. The hole doping in the iridate is roughly equivalent to the electron doping in cuprates. Nevertheless, we suggest that the symmetrybreaking phenomena in the hidden-order phase of Rhdoped $\mathrm{Sr}_{2} \mathrm{IrO}_{4}$ bear a striking resemblance to those in the pseudogap phase in hole-doped cuprates. In particular, the emergence of rotational symmetry breaking is a remarkable common feature of two different Mott insulating systems. Thus, detailed comparisons of the nematicity between the cuprates and the iridate could reveal important information on the peculiar electronic properties of both systems.
It has been reported that the nematic director in $\mathrm{YBa}_{2} \mathrm{Cu}_{3} \mathrm{O}_{6+\delta}$ and $(\mathrm{Bi}, \mathrm{Pb})_{2} \mathrm{Sr}_{2} \mathrm{CaCu}_{2} \mathrm{O}_{8+\delta}$ orients along the $\mathrm{Cu}-\mathrm{O}-\mathrm{Cu}$ bond direction $[63,74]$, while it orients along the diagonal direction of the $\mathrm{CuO}_{2}$ square lattice in $\mathrm{HgBa}_{2} \mathrm{CuO}_{4+\delta}$ [64]. Although the difference of the nematic direction among cuprates remains an open question, it may be due to the number of $\mathrm{CuO}_{2}$ planes in the unit cell [21]. Therefore, the comparison between the monolayer cuprate $\mathrm{HgBa}_{2} \mathrm{CuO}_{4+\delta}$ and the single layered iridate $\mathrm{Sr}_{2} \mathrm{IrO}_{4}$ is more relevant. In $\mathrm{HgBa}_{2} \mathrm{CuO}_{4+\delta}$, polarized neutron diffraction experiments have revealed an unusual $\boldsymbol{q}=0$ magnetic order below the pseudogap temperature, which has been discussed in terms of loop-current-like magnetism breaking both time-reversal and parity symmetries $[19,75,76]$. The in-plane torque magnetometry in $\mathrm{HgBa}_{2} \mathrm{CuO}_{4+\delta}$ reported that the nematic director orients along the diagonal direction of the $\mathrm{CuO}_{2}$ square lattice, whereas neutron scattering experiments reported that the loop currents in $\mathrm{HgBa}_{2} \mathrm{CuO}_{4+\delta}$ are not only oriented diagonally but are also out of plane [76]. Although both in-plane torque and nematic susceptibility measurements are insensitive to the out-of-plane properties, we point out that the nematic director in the hidden-order phase of $\mathrm{Sr}_{2} \mathrm{IrO}_{4}$ is rotated $45^{\circ}$ from that in the pseudogap state of $\mathrm{HgBa}_{2} \mathrm{CuO}_{4+\delta}$ in the basal plane. Therefore, if the loopcurrent-like magnetism is an origin of the nematicity, the loop-current pattern of $\mathrm{Sr}_{2} \mathrm{IrO}_{4}$, which is illustrated in Fig. 4(b), must be different from that of $\mathrm{HgBa}_{2} \mathrm{CuO}_{4+\delta}$. Our results show that the intra-unit-cell loop current flows only in one of the diagonal directions in the $\mathrm{IrO}_{4}$ square in both pure and $\mathrm{Rh}$-doped $\mathrm{Sr}_{2} \mathrm{IrO}_{4}$ [15-17].

The interpretation of the twofold oscillations in the AFM-ordered states is rather complicated. The rapid suppression of the amplitude of twofold oscillations with $B_{2 g}$ symmetry below $T_{N}^{S}$ may be due to the segmentation of the domain structure by short-range magnetic order. A further detailed study is required to clarify how the shortrange magnetic order influences the anapole moment. As shown in Figs. 8(a)-8(c), two-fold oscillations with $B_{1 g}$ symmetry appear below the long-range AFM-ordering temperatures, which may be due to the coupling between the pseudospin and the lattice [77].

\section{CONCLUSIONS}

In summary, we measured the in-plane magnetic torque and elastoresistance in a spin-orbit coupled Mott insulator $\mathrm{Sr}_{2}\left(\operatorname{Ir}_{1-x} \mathrm{Rh}_{x}\right) \mathrm{O}_{4}$. The measurements of in-plane anisotropy of the magnetic susceptibility reveal the emergence of the nematic phase with broken rotation symmetry distinctly above the Néel transition. On the other hand, nematic susceptibility exhibits no diverging behavior when approaching the nematic critical point. These results provide bulk evidence for the odd-parity hidden-order state suggested by the SHG. The results, along with the report of polarized neutron diffraction, suggest that the hidden-order 
phase is in an anapole state, which is composed of a polar (magnetic) toroidal dipole induced by persistent loop currents. These results may bear a resemblance to the pseudogap state of underdoped cuprates, but the nematic director of $\mathrm{Sr}_{2}\left(\mathrm{Ir}_{1-x} \mathrm{Rh}_{x}\right) \mathrm{O}_{4}$ is rotated $45^{\circ}$ from that of the monolayer cuprate $\mathrm{HgBa}_{2} \mathrm{CuO}_{4+\delta}$, implying the difference of the loop-current patterns between the two systems. The remarkable common features of the nematicity and distinct differences of the nematic director may be key to understanding the symmetry-breaking phenomena in the hiddenorder state of the iridate and the pseudogap state of the cuprates.

\section{ACKNOWLEDGMENTS}

We thank T. Kimura, H. Kontani, and E.-G. Moon for discussions. This work is supported by Grants-in-Aid for Scientific Research (KAKENHI) (No. JP18H01177, No. JP18H01178, No. JP18H01180, No. JP18H05227, No. JP18K13492, No. JP19H00649, No. JP20H02600, No. JP20H05159, and No. JP20K21139) and on Innovative Areas "Quantum Liquid Crystals" (No. JP19H05824) from Japan Society for the Promotion of Science (JSPS), and JST CREST (JPMJCR19T5). G. C. acknowledges NSF support via Grant No. DMR 1903888.

[1] N. A Spaldin, M. Fiebig, and M. Mostovoy, The Toroidal Moment in Condensed-Matter Physics and Its Relation to the Magnetoelectric Effect, J. Phys. Condens. Matter 20, 434203 (2008).

[2] S. Nanz, Multipole Expansion of the Potentials: Toroidal Multipole Moments in Classical Electrodynamics-An Analysis of Their Emergence and Physical Significance (Springer Spektrum, Wiesbaden, 2016).

[3] S. Gnewuch and E. E. Rodriguez, The Fourth Ferroic Order: Current Status on Ferrotoroidic Materials, J. Solid State Chem. 271, 175 (2019).

[4] V. M. Dubovik, L. A. Tosunyan, and V. V. Tugushev, Axial Toroidal Moments in Electrodynamics and Solid-State Physics, JETP Lett. 63, 344 (1986).

[5] V. M. Dubovik and V. V. Tugushev, Toroid Moments in Electrodynamics and Solid-State Physics, Phys. Rep. 187, 145 (1990).

[6] A. K. Yadav, C. T. Nelson, S. L. Hsu, Z. Hong, J. D. Clarkson, C. M. Schlepütz, A. R. Damodaran, P. Shafer, E. Arenholz, L. R. Dedon, D. Chen, A. Vishwanath, A. M. Minor, L. Q. Chen, J. F. Scott, L. W. Martin, and R. Ramesh, Observation of Polar Vortices in Oxide Superlattices, Nature (London) 530, 198 (2016).

[7] W. Jin, E. Drueke, S. Li, A. Admasu, R. Owen, M. Day, K. Sun, S.-W. Cheong, and L. Zhao, Observation of a Ferrorotational Order Coupled with Second-Order Nonlinear Optical Fields, Nat. Phys. 16, 42 (2020).

[8] A. Nakano, T. Hasegawa, S. Tamura, N. Katayama, S. Tsutsui, and H. Sawa, Antiferroelectric Distortion with
Anomalous Phonon Softening in the Excitonic Insulator, $\mathrm{Ta}_{2} \mathrm{NiSe}_{5}$, Phys. Rev. B 98, 045139 (2018).

[9] B. B. Van Aken, J.-P. Rivera, H. Schmid, and M. Fiebig, Observation of Ferrotoroidic Domains, Nature (London) 449, 702 (2007).

[10] V. Scagnoli, U. Staub, Y. Bodenthin, R. A. de Souza, M. García-Fernández, M. Garganourakis, A. T. Boothroyd, D. Prabhakaran, and S. W. Lovesey, Observation of Orbital Currents in $\mathrm{CuO}$, Science 332, 696 (2011).

[11] S. Di Matteo and M. R. Norman, Orbital Currents, Anapoles, and Magnetic Quadrupoles in $\mathrm{CuO}$, Phys. Rev. B 85, 235143 (2012).

[12] C. M. Varma, Theory of the Pseudogap State of the Cuprates, Phys. Rev. B 73, 155113 (2006).

[13] C. M. Varma, Pseudogap in Cuprates in the LoopCurrent Ordered State, J. Phys. Condens. Matter 26, 505701 (2014).

[14] S. S. Pershoguba, K. Kechedzhi, and V. M. Yakovenko, Proposed Chiral Texture of the Magnetic Moments of Unit-Cell Loop Currents in the Pseudogap Phase of Cuprate Superconductors, Phys. Rev. Lett. 111, 047005 (2013); S. S. Pershoguba, K. Kechedzhi, and V. M. Yakovenko, Erratum: Proposed Chiral Texture of the Magnetic Moments of Unit-Cell Loop Currents in the Pseudogap Phase of Cuprate, Phys. Rev. Lett. 113, 129901 (2014).

[15] S. Chatterjee, S. Sachdev, and M. S. Scheurer, Intertwining Topological Order and Broken Symmetry in a Theory of Fluctuating Spin-Density Waves, Phys. Rev. Lett. 119, 227002 (2017).

[16] M. S. Scheurer and S. Sachdev, Orbital Currents in Insulating and Doped Antiferromagnets, Phys. Rev. B 98, 235126 (2018).

[17] S. Sachdev, H. D. Scammell, M. S. Scheurer, and G. Tarnopolsky, Gauge Theory for the Cuprates Near Optimal Doping, Phys. Rev. B 99, 054516 (2019).

[18] B. Fauqué, Y. Sidis, V. Hinkov, S. Pailheś, C. T. Lin, X. Chaud, and P. Bourges, Magnetic Order in the Pseudogap Phase of High- $T_{c}$ Superconductors, Phys. Rev. Lett. 96, 197001 (2006).

[19] Y. Li, V. Balédent, N. Barišić, Y. Cho, B. Fauqué, Y. Sidis, G. Yu, X. Zhao, P. Bourges, and M. Greven, Unusual Magnetic Order in the Pseudogap Region of the Superconductor $\mathrm{HgBa}_{2} \mathrm{CuO}_{4+\delta}$, Nature (London) 455, 372 (2008).

[20] S. De Almeida-Didry, Y. Sidis, V. Baledent, F. Giovannelli, I. Monot-Laffez, and P. Bourges, Evidence for Intra-UnitCell Magnetic Order in $\mathrm{Bi}_{2} \mathrm{Sr}_{2} \mathrm{CaCu}_{2} \mathrm{O}_{8+\delta}$, Phys. Rev. B 86, 020504(R) (2012).

[21] L. Mangin-Thro, Y. Li, Y. Sidis, and P. Bourges, $a-b$ Anisotropy of the Intra-Unit-Cell Magnetic Order in $\mathrm{YBa}_{2} \mathrm{Cu}_{3} \mathrm{O}_{6.6}$, Phys. Rev. Lett. 118, 097003 (2017).

[22] T. P. Croft, E. Blackburn, J. Kulda, R. Liang, D. A. Bonn, W. N. Hardy, and S. M. Hayden, No Evidence for Orbital Loop Currents in Charge-Ordered $\mathrm{YBa}_{2} \mathrm{Cu}_{3} \mathrm{O}_{6+x}$ from Polarized Neutron Diffraction, Phys. Rev. B 96, 214504 (2017).

[23] P. Bourges, Y. Sidis, and L. Mangin-Thro, Comment on "No Evidence for Orbital Loop Currents in Charge-Ordered $\mathrm{YBa}_{2} \mathrm{Cu}_{3} \mathrm{O}_{6+x}$ from Polarized Neutron Diffraction, Phys. Rev. B 98, 016501 (2018). 
[24] T. P. Croft, E. Blackburn, J. Kulda, R. Liang, D. A. Bonn, W. N. Hardy, and S. M. Hayden, Reply to "Comment on 'No Evidence for Orbital Loop Currents in Charge-Ordered $\mathrm{YBa}_{2} \mathrm{Cu}_{3} \mathrm{O}_{6+x}$ from Polarized Neutron Diffraction,"” Phys. Rev. B 98, 016502 (2018).

[25] B. J. Kim, H. Jin, S. J. Moon, J.-Y. Kim, B.-G. Park, C. S. Leem, J. Yu, T. W. Noh, C. Kim, S.-J. Oh, J.-H. Park, V. Durairaj, G. Cao, and E. Rotenberg, Novel $J_{\text {eff }}=1 / 2$ Mott State Induced by Relativistic Spin-Orbit Coupling in $\mathrm{Sr}_{2} \mathrm{IrO}_{4}$, Phys. Rev. Lett. 101, 076402 (2008).

[26] J. G. Rau, E. K. -H. Lee, and H. Y. Kee, Spin-Orbit Physics Giving Rise to Novel Phases in Correlated Systems: Iridates and Related Materials, Annu. Rev. Condens. Matter Phys. 7, 195 (2016).

[27] G. Cao and P. Schlottmann, The Challenge of Spin-OrbitTuned Ground States in Iridates: A Key Issues Review, Rep. Prog. Phys. 81, 042502 (2018).

[28] J. Bertinshaw, Y. K. Kim, G. Khaliullin, and B. J. Kim, Square Lattice Iridates, Annu. Rev. Condens. Matter Phys. 10, 315 (2019).

[29] J. Dai, E. Calleja, G. Cao, and K. McElroy, Local Density of States Study of a Spin-Orbit-Coupling Induced Mott Insulator $\mathrm{Sr}_{2} \mathrm{IrO}_{4}$, Phys. Rev. B 90, 041102(R) (2014).

[30] M. K. Crawford, M. A. Subramanian, R. L. Harlow, J. A. Fernandez-Baca, Z. R. Wang, and D. C. Johnston, Structural and Magnetic Studies of $\mathrm{Sr}_{2} \mathrm{IrO}_{4}$, Phys. Rev. B 49, 9198 (1994).

[31] C. Dhital, T. Hogan, Z. Yamani, C. de la Cruz, X. Chen, S. Khadka, Z. Ren, and S. D. Wilson, Neutron Scattering Study of Correlated Phase Behavior in $\mathrm{Sr}_{2} \mathrm{IrO}_{4}$, Phys. Rev. B 87, 144405 (2013).

[32] F. Ye, S. Chi, B. C. Chakoumakos, J. A. Fernandez-Baca, T. Qi, and G. Cao, Magnetic and Crystal Structures of $\mathrm{Sr}_{2} \mathrm{IrO}_{4}$ : A Neutron Diffraction Study, Phys. Rev. B 87, 140406(R) (2013).

[33] D. H. Torchinsky, H. Chu, L. Zhao, N. B. Perkins, Y. Sizyuk, T. Qi, G. Cao, and D. Hsieh, Structural Distortion-Induced Magnetoelastic Locking in $\mathrm{Sr}_{2} \mathrm{IrO}_{4}$ Revealed through Nonlinear Optical Harmonic Generation, Phys. Rev. Lett. 114, 096404 (2015).

[34] B. J. Kim, H. Ohsumi, T. Komesu, S. Sakai, T. Morita, H. Takagi, and T. Arima, Phase-Sensitive Observation of a Spin-Orbital Mott State in $\mathrm{Sr}_{2} \mathrm{IrO}_{4}$, Science 323, 1329 (2009).

[35] S. Boseggia, H. C. Walker, J. Vale, R. Springell, Z. Feng, R. S. Perry, M. M. Sala, H. M. Rønnow, S. P. Collins, and D. F. McMorrow, Locking of Iridium Magnetic Moments to the Correlated Rotation of Oxygen Octahedra in $\mathrm{Sr}_{2} \mathrm{IrO}_{4}$ Revealed by X-Ray Resonant Scattering, J. Phys. Condens. Matter 25, 422202 (2013).

[36] J. Kim, D. Casa, M. H. Upton, T. Gog, Y.-J. Kim, J. F. Mitchell, M. van Veenendaal, M. Daghofer, J. van den Brink, G. Khaliullin, and B. J. Kim, Magnetic Excitation Spectra of $\mathrm{Sr}_{2} \mathrm{IrO}_{4}$ Probed by Resonant Inelastic X-Ray Scattering: Establishing Links to Cuprate Superconductors, Phys. Rev. Lett. 108, 177003 (2012).

[37] F. Wang and T. Senthil, Twisted Hubbard Model for $\mathrm{Sr}_{2} \mathrm{IrO}_{4}$ : Magnetism and Possible High Temperature Superconductivity, Phys. Rev. Lett. 106, 136402 (2011).
[38] M. Ge, T. F. Qi, O. B. Korneta, D. E. De Long, P. Schlottmann, W. P. Crummett, and G. Cao, Lattice-Driven Magnetoresistivity and Metal-Insulator Transition in Single-Layered Iridates, Phys. Rev. B 84, 100402(R) (2011).

[39] X. Chen, T. Hogan, D. Walkup, W. Zhou, M. Pokharel, M. Yao, W. Tian, T. Z. Ward, Y. Zhao, D. Parshall, C. Opeil, J. W. Lynn, V. Madhavan, and S. D. Wilson, Influence of Electron Doping on the Ground State of $\left(\mathrm{Sr}_{1-x} \mathrm{La}_{x}\right)_{2} \mathrm{IrO}_{4}$, Phys. Rev. B 92, 075125 (2015).

[40] Y. K. Kim, O. Krupin, J. D. Denlinger, A. Bostwick, E. Rotenberg, Q. Zhao, J. F. Mitchell, J. W. Allen, and B. J. Kim, Fermi Arcs in a Doped Pseudospin-1/2 Heisenberg Antiferromagnet, Science 345, 187 (2014).

[41] Y. K. Kim, N. H. Sung, J. D. Denlinger, and B. J. Kim, Observation of a d-Wave Gap in Electron-Doped $\mathrm{Sr}_{2} \mathrm{IrO}_{4}$, Nat. Phys. 12, 37 (2016).

[42] V. Brouet, J. Mansart, L. Perfetti, C. Piovera, I. Vobornik, P. Le Fèvre, F. Bertran, S. C. Riggs, M. C. Shapiro, P. GiraldoGallo, and I. R. Fisher, Transfer of Spectral Weight Across the Gap of $\mathrm{Sr}_{2} \mathrm{IrO}_{4}$ Induced by La Doping, Phys. Rev. B 92 , 081117(R) (2015).

[43] A. de la Torre, S. M. Walker, F. Y. Bruno, S. Riccó, Z. Wang, I. G. Lezama, G. Scheerer, G. Giriat, D. Jaccard, C. Berthod, T. K. Kim, M. Hoesch, E. C. Hunter, R. S. Perry, A. Tamai, and F. Baumberger, Collapse of the Mott Gap and Emergence of a Nodal Liquid in Lightly Doped $\mathrm{Sr}_{2} \mathrm{IrO}_{4}$, Phys. Rev. Lett. 115, 176402 (2015).

[44] Y. J. Yan, M. Q. Ren, H. C. Xu, B. P. Xie, R. Tao, H. Y. Choi, N. Lee, Y. J. Choi, T. Zhang, and D. L. Feng, Electron-Doped $\mathrm{Sr}_{2} \mathrm{IrO}_{4}$ : An Analogue of Hole-Doped Cuprate Superconductors Demonstrated by Scanning Tunneling Microscopy, Phys. Rev. X 5, 041018 (2015).

[45] I. Battisti, K. M. Bastiaans, V. Fedoseev, A. de la Torre, N. Iliopoulos, A. Tamai, E. C. Hunter, R. S. Perry, J. Zaanen, F. Baumberger, and M.P. Allan, Universality of Pseudogap and Emergent Order in Lightly Doped Mott Insulators, Nat. Phys. 13, 21 (2017).

[46] H. Watanabe, T. Shirakawa, and S. Yunoki, Monte Carlo Study of an Unconventional Superconducting Phase in Iridium Oxide $J_{\mathrm{eff}}=1 / 2$ Mott Insulators Induced by Carrier Doping, Phys. Rev. Lett. 110, 027002 (2013).

[47] Y. Yang, W.-S. Wang, J.-G. Liu, H. Chen, J.-H. Dai, and Q.-H. Wang, Superconductivity in Doped $\mathrm{Sr}_{2} \mathrm{IrO}_{4}$ : A Functional Renormalization Group Study, Phys. Rev. B 89, 094518 (2014).

[48] Z. Y. Meng, Y. B. Kim, and H.-Y. Kee, Odd-Parity Triplet Superconducting Phase in Multiorbital Materials with a Strong Spin-Orbit Coupling: Application to Doped $\mathrm{Sr}_{2} \mathrm{IrO}_{4}$, Phys. Rev. Lett. 113, 177003 (2014).

[49] T. F. Qi, O. B. Korneta, L. Li, K. Butrouna, V. S. Cao, X. Wan, P. Schlottmann, R. K. Kaul, and G. Cao, Spin-Orbit Tuned Metal-Insulator Transitions in Single-Crystal $\mathrm{Sr}_{2}\left(\operatorname{Ir}_{1-x} \mathrm{Rh}_{x}\right) \mathrm{O}_{4}(0 \leq x \leq 1)$, Phys. Rev. B 86, 125105 (2012).

[50] F. Ye, X. Wang, C. Hoffmann, J. Wang, S. Chi, M. Matsuda, B. C. Chakoumakos, J. A. Fernandez-Baca, and G. Cao, Structure Symmetry Determination and Magnetic Evolution in $\mathrm{Sr}_{2}\left(\mathrm{Ir}_{1-x} \mathrm{Rh}_{x}\right) \mathrm{O}_{4}$, Phys. Rev. B 92, 201112(R) (2015). 
[51] J. P. Clancy, A. Lupascu, H. Gretarsson, Z. Islam, Y. F. Hu, D. Casa, C. S. Nelson, S. C. LaMarra, G. Cao, and Y.-J. Kim, Dilute Magnetism and Spin-Orbital Percolation Effects in $\mathrm{Sr}_{2}\left(\mathrm{Ir}_{1-x} \mathrm{Rh}_{x}\right) \mathrm{O}_{4}$, Phys. Rev. B 89, 054409 (2014).

[52] Y. Cao, Q. Wang, J. A. Waugh, T. J. Reber, H. Li, X. Zhou, S. Parham, S.-R. Park, N. C. Plumb, E. Rotenberg, A. Bostwick, J. D. Denlinger, T. Qi, M. A. Hermele, G. Cao, and D. S. Dessau, Hallmarks of the Mott-Metal Crossover in the Hole-Doped Pseudospin-1/2 Mott Insulator $\mathrm{Sr}_{2} \mathrm{IrO}_{4}$, Nat. Commun. 7, 11367 (2016).

[53] A. Louat, F. Bert, L. Serrier-Garcia, F. Bertran, P. Le Fèvre, J. Rault, and V. Brouet, Formation of an Incoherent Metallic State in Rh-Doped $\mathrm{Sr}_{2} \mathrm{IrO}_{4}$, Phys. Rev. B 97, 161109(R) (2018).

[54] B. Keimer, S. A. Kivelson, M. R. Norman, S. Uchida, and J. Zaanen, From Quantum Matter to High-Temperature Superconductivity in Copper Oxides, Nature (London) 518, 179 (2015).

[55] L. Zhao, D. H. Torchinsky, H. Chu, V. Ivanov, R. Lifshitz, R. Flint, T. Qi, G. Cao, and D. Hsieh, Evidence of an OddParity Hidden Order in a Spin-Orbit Coupled Correlated Iridate, Nat. Phys. 12, 32 (2016).

[56] L. Zhao, C. A. Belvin, R. Liang, D. A. Bonn, W. N. Hardy, N.P. Armitage, and D. Hsieh, A Global InversionSymmetry-Broken Phase Inside the Pseudogap Region of $\mathrm{YBa}_{2} \mathrm{Cu}_{3} \mathrm{O}_{y}$, Nat. Phys. 13, 250 (2017).

[57] J. W. Harter, Z. Y. Zhao, J.-Q. Yan, D. G. Mandrus, and D. Hsieh, A Parity-Breaking Electronic Nematic Phase Transition in the Spin-Orbit Coupled Metal $\mathrm{Cd}_{2} \mathrm{Re}_{2} \mathrm{O}_{7}$, Science 356, 295 (2017).

[58] J. Jeong, Y. Sidis, A. Louat, V. Brouet, and P. Bourges, Time-Reversal Symmetry Breaking Hidden Order in $\mathrm{Sr}_{2}(\mathrm{Ir}, \mathrm{Rh}) \mathrm{O}_{4}$, Nat. Commun. 8, 15119 (2017).

[59] C. Tan, Z. F. Ding, J. Zhang, Z. H. Zhu, O. O. Bernal, P. C. Ho, A. D. Hillier, A. Koda, H. Luetkens, G. D. Morris, D. E. MacLaughlin, and L. Shu, Slow Magnetic Fluctuations and Critical Slowing Down in $\mathrm{Sr}_{2}\left(\mathrm{Ir}_{1-x} \mathrm{Rh}_{x}\right) \mathrm{O}_{4}$, Phys. Rev. B 101, 195108 (2020).

[60] J. Zhang, Z. Ding, C. Tan, K. Huang, O. O. Bernal, P.-C. Ho, G. D. Morris, A. D. Hillier, P. K. Biswas, S. P. Cottrell, H. Xiang, X. Yao, D. E. MacLaughlin, and L. Shu, Discovery of Slow Magnetic Fluctuations and Critical Slowing Down in the Pseudogap Phase of $\mathrm{YBa}_{2} \mathrm{Cu}_{3} \mathrm{O}_{y}$, Sci. Adv. 4, eaao5235 (2018).

[61] R. Okazaki, T. Shibauchi, H. J. Shi, Y. Haga, T. D. Matsuda, E. Yamamoto, Y. Onuki, H. Ikeda, and Y. Matsuda, Rotational Symmetry Breaking in the Hidden-Order Phase of $\mathrm{URu}_{2} \mathrm{Si}_{2}$, Science 331, 439 (2011).

[62] S. Kasahara, H. J. Shi, K. Hashimoto, S. Tonegawa, Y. Mizukami, T. Shibauchi, K. Sugimoto, T. Fukuda, T. Terashima, A. H. Nevidomskyy, and Y. Matsuda, Ectronic Nematicity above the Structural and Superconducting Transition in $\mathrm{BaFe}_{2}\left(\mathrm{As}_{1-x} \mathrm{P}_{x}\right)_{2}$, Nature (London) 486, 382 (2012).

[63] Y. Sato, S. Kasahara, H. Murayama, Y. Kasahara, E.-G. Moon, T. Nishizaki, T. Loew, J. Porras, B. Keimer, T. Shibauchi, and Y. Matsuda, Thermodynamic Evidence for a Nematic Phase Transition at the Onset of the Pseudogap in $\mathrm{YBa}_{2} \mathrm{Cu}_{3} \mathrm{O}_{y}$, Nat. Phys. 13, 1074 (2017).

[64] H. Murayama, Y. Sato, R. Kurihara, S. Kasahara, Y. Mizukami, Y. Kasahara, H. Uchiyama, A. Yamamoto,
E. G. Moon, J. Cai, J. Freyermuth, M. Greven, T. Shibauchi, and Y. Matsuda, Diagonal Nematicity in the Pseudogap Phase of $\mathrm{HgBa}_{2} \mathrm{CuO}_{4+\delta}$, Nat. Commun. 10, 3282 (2019).

[65] J.-H. Chu, H.-H. Kuo, J. G. Analytis, and I. R. Fisher, Divergent Nematic Susceptibility in an Iron Arsenide Superconductor, Science 337, 710 (2012).

[66] H.-H. Kuo, J.-H. Chu, J. C Palmstrom, S. A Kivelson, and I. R Fisher, Ubiquitous Signatures of Nematic Quantum Criticality in Optimally Doped Fe-Based Superconductors, Science 352, 958 (2016).

[67] M. C. Shapiro, A. T. Hristov, J. C. Palmstrom, J.-H. Chu, and I. R. Fisher, Measurement of the $B_{1 g}$ and $B_{2 g}$ Components of the Elastoresistivity Tensor for Tetragonal Materials via Transverse Resistivity Configurations, Rev. Sci. Instrum. 87, 063902 (2016).

[68] S. Hosoi, K. Matsuura, K. Ishida, H. Wang, Y. Mizukami, T. Watashige, S. Kasahara, Y. Matsuda, and T. Shibauchi, Nematic Quantum Critical Point without Magnetism in FeSe $_{1-x} \mathrm{~S}_{x}$ Superconductors, Proc. Natl. Acad. Sci. U.S.A. 113, 8139 (2016).

[69] K. Ishida, M. Tsujii, S. Hosoi, Y. Mizukami, S. Ishida, A. Iyo, H. Eisaki, T. Wolf, K. Grube, H. v. Loehneysen, R. M. Fernandes, and T. Shibauchi, Novel Electronic Nematicity in Heavily Hole-Doped Iron Pnictide Superconductors, Proc. Natl. Acad. Sci. U.S.A. 117, 6424 (2020).

[70] K. Ishida, S. Hosoi, Y. Teramoto, T. Usui, Y. Mizukami, K. Itaka, Y. Matsuda, T. Watanabe, and T. Shibauchi, Divergent Nematic Susceptibility Near the Pseudogap Critical Point in a Cuprate Superconductor, J. Phys. Soc. Jpn. 89, 064707 (2020).

[71] L. Fruchter, D. Colson, and V. Brouet, Magnetic Critical Properties and Basal-Plane Anisotropy of $\mathrm{Sr}_{2} \mathrm{IrO}_{4}$, J. Phys. Condens. Matter 28, 126003 (2016).

[72] M. Nauman, Y. Hong, T. Hussain, M. S. Seo, S. Y. Park, N. Lee, Y. J. Choi, W. Kang, and Y. Jo, In-Plane Magnetic Anisotropy in Strontium Iridate $\mathrm{Sr}_{2} \mathrm{IrO}_{4}$, Phys. Rev. B 96, 155102 (2017)

[73] T. Shibauchi, T. Hanaguri, and Y. Matsuda, Exotic Superconducting States in FeSe-Based Materials, J. Phys. Soc. Jpn. 89, 102002 (2020).

[74] N. Auvray, B. Loret, S. Benhabib, M. Cazayous, R. D. Zhong, J. Schneeloch, G. D. Gu, A. Forget, D. Colson, I. Paul, A. Sacuto, and Y. Gallais, Nematic Fluctuations in the Cuprate Superconductor $\mathrm{Bi}_{2} \mathrm{Sr}_{2} \mathrm{CaCu}_{2} \mathrm{O}_{8+\delta}$, Nat. Commun. 10, 5209 (2019).

[75] V. M. Yakovenko, Tilted Loop Currents in Cuprate Superconductors, Physica B (Amsterdam) 460, 159 (2015).

[76] Y. Tang, L. Mangin-Thro, A. Wildes, M. K. Chan, C. J. Dorow, J. Jeong, Y. Sidis, M. Greven, and P. Bourges, Orientation of the Intra-Unit-Cell Magnetic Moment in the High- $T_{c}$ Superconductor $\mathrm{HgBa}_{2} \mathrm{CuO}_{4+\delta}$, Phys. Rev. B 98, 214418 (2018).

[77] J. Porras, J. Bertinshaw, H. Liu, G. Khaliullin, N. H. Sung, J.-W. Kim, S. Francoual, P. Steffens, G. Deng, M. M. Sala, A. Efimenko, A. Said, D. Casa, X. Huang, T. Gog, J. Kim, B. Keimer, and B. J. Kim, Pseudospin-Lattice Coupling in the Spin-Orbit Mott Insulator $\mathrm{Sr}_{2} \mathrm{IrO}_{4}$, Phys. Rev. B 99, 085125 (2019). 